\title{
The population genetics of the self- incompatibility polymorphism in Papaver rhoeas. VIII. Sampling effects as a possible cause of unequal allele frequencies
}

\author{
M. J. LAWRENCE*, S. O'DONNELL, M. D. LANE \& D. F. MARSHALL \\ Wolfson Laboratory for Plant Molecular Biology, School of Biological Sciences, University of Birmingham, Birmingham \\ $B 152 T T$, U.K.
}

\begin{abstract}
This paper considers the hypothesis that the unequal $S$-allele frequencies in the British populations of Papaver rhoeas we have examined are caused by a sampling effect over and above that due to drift. The results obtained by simulating the polymorphism on the computer show that it takes between 70 and 80 non-overlapping generations before stochastic equilibrium is achieved with respect to allele frequency in a population of size 3720 containing 31 alleles, both when the frequencies of the alleles are initially as unequal as they were in the R106 sample (Campbell \& Lawrence, 1981b) and when these frequencies are exactly equal. These results show that the strength of the frequency-dependent selection that maintains the polymorphism becomes very considerably attenuated as the number of alleles in the population increases. A review of the relevant genetical and ecological evidence suggests that the size of the British populations is considerably larger than the minimum required to maintain the number of alleles they are estimated to contain, so that opportunities for sampling effects to perturb the frequencies of these alleles appear to be limited. The results obtained from an investigation of the reproductive biology of the species, however, show that the distribution of progeny size is markedly non-random. It is possible that this may be the cause of the unequal allele frequencies observed in the the British populations.
\end{abstract}

Keywords: drift, effective population size, Papaver rhoeas, population genetics, reproductive biology, self-incompatibility.

\section{Introduction}

We have reported, in previous papers, that the results obtained from a survey of the number and frequency of $S$-alleles in samples drawn at random from three British natural populations of Papaver rhoeas showed that, contrary to expectation, the frequencies of the alleles in each of these samples were significantly unequal (Campbell \& Lawrence, 1981b; Lawrence \& O'Donnell, 1981). In attempting to account for this unexpected observation, we proposed two alternative hypotheses. Firstly, that some of the alleles in the population from which these samples were drawn were subject to an extra effect of selection, over and above that concerned with incompatibility, due either to a pleiotropic property of these alleles or to their linkage

\footnotetext{
${ }^{*}$ Correspondence.
}

to another gene that is the chief target of this extra effect of selection. Secondly, that the unequal allele frequencies are caused by a sampling effect, over and above that caused by drift, of a kind to be expected in a species occurring in disturbed habitats.

At the time they were proposed, there was insufficient evidence to decide which of these hypotheses provided the more likely explanation of our observation. For reasons given in an earlier paper (Lawrence et al., 1993), however, it is now possible effectively to rule out the version of the first hypothesis which supposes that an extra effect of selection is due to a pleiotropic property of some of the $S$-alleles. Hence, if the unequal allele frequencies in the populations from which these samples have been drawn are, in fact, caused by an extra effect of selection, it now seems more likely that this is due to linkage of the $S$-gene to another that is the chief target of this selection, rather than to a pleiotropic property of some of the alleles in these populations. 
While, formally, these two hypotheses are quite different, their effects on the polymorphism are, nevertheless, similar. Thus, for reasons given in a previous paper (Lawrence et al., 1993), on both, different alleles are expected to occur at a relatively high frequency in different populations, both envisage that the population is in a state of disequilibrium and that, accordingly, on both, in the absence of further perturbation, the population will, in time, achieve equilibrium with respect to the alleles it contains. For these reasons, it is unlikely that any purely observational data obtained from surveys of the polymorphism in further populations of the species will allow us to discriminate between these hypotheses. We need, therefore, to consider what other kinds of evidence might allow us to do this.

Now, in principle, it should be possible to test the first hypothesis by examining segregation ratios in fullsib families that are segregating for an allele that occurs at a relatively high frequency in the population from which the parents of these families originated and three alleles that occur at a relatively low frequency in that population. This is a possibility to which we shall turn in the following paper (Lawrence \& Franklin-Tong, 1994). There is, however, no obvious experimental test of the second hypothesis. Furthermore, while a considerable amount of attention has been devoted to the attainment of equilibrium with respect to the number of alleles in a population of given size (Wright, 1939, 1960, 1964; Fisher, 1958; Moran, 1962; Ewens, 1964; Crosby, 1966; Ewens \& Ewens, 1966; Kimura \& Maruyama, 1966; Mayo, 1966), the attainment of equilibrium with respect to the frequencies of these alleles has received less attention. Hence, this theory, much of which concerned the validity of the approximations used by Wright (1939) in his analysis of the properties of the polymorphism and which was, in any case, largely directed towards the Oenothera problem (Emerson, 1939), provides little direct guidance on how many generations it might take to attain equilibrium in the absence of further perturbation. It seemed worthwhile, therefore, to attempt to obtain some information about this question by simulating the polymorphism on the computer. In the present paper, we report the results obtained from a preliminary investigation of this problem. The simulation program was also used, for reasons that will emerge, to assess the power of the modified $\chi^{2}$ that we have employed to test the null hypothesis that the $S$-alleles in the populations from which the samples we have examined have been drawn were equally frequent (Campbell \& Lawrence, 1981b; Lawrence \& O'Donnell, 1981). Finally, we discuss the results obtained from an investigation of some aspects of the population biology of the species in an attempt to identify the kind of environmental perturbation which might have caused the unequal allele frequencies.

\section{Simulation program}

The program, written in FORTRAN, was similar to that used by Crosby (1966) in his investigation of the Oenothera problem. Each run of the program was initiated with a population of desired size containing a specified set of genotypes giving a desired set of allele frequencies. The genotypes in each generation were produced from those of the preceding one in the following way. Two plants were chosen at random from the population, the first being regarded as the female parent. If the two plants were fully compatible, one of the female and one of the male alleles were chosen at random to form a zygote. If the two plants were halfcompatible, the compatible male allele was combined with one of the female alleles chosen at random. If the two plants were incompatible, the female was retained, the male was returned to the population and a second male chosen at random. This procedure was repeated to give the desired number of genotypes. Generations were non-overlapping and the size of the population was held constant throughout each run. Mutation was not simulated. The NAG library routine G05CAF was used to generate random numbers and the simulations reported here were obtained by running the program on the University of Birmingham's mainframe computer. The program, when first written, was tested by simulating the annual models (R1-R4) of Crosby (1966); in every case, it gave similar results to those he obtained. Insofar that the program simulates random mating in a panmictic population it is, perhaps, not entirely realistic although in these circumstances the effective size of the population, $N_{\mathrm{e}}$, is conveniently equal to its actual size, $N$. This lack of realism, however, is of little importance to our present concerns.

\section{Results of the simulation}

Two populations were investigated, the genotype frequencies in the first being identical to those of the R106 sample in which the allele frequencies were the most unequal of the three samples we have examined (Campbell \& Lawrence, 1981b). The initial number of alleles in the population was, therefore, 31 . Because we wished to investigate the effect of drift on the frequencies of these alleles without losing any during the course of a run, each of the 51 genotype of the R106 sample was replicated 73 times to give a population of size 3723 , a size which appeared sufficiently large, from Wright's (1939) Fig. 2, to achieve this objec- 
Fig. 1 The approach towards stochastic equilibrium of allele frequency. The upper curves show two independent runs of a population of size 3723 containing 31 alleles whose initial frequencies were the same as those of the R106 sample. The lower curves show two independent runs of a population of size 3720 , also containing 31 alleles, but whose initial frequencies were exactly equal. The graph shows the variance of allele frequency plotted against generation.

Fig. 2 As for Fig. 1 but showing the average of ten independent runs of each population.
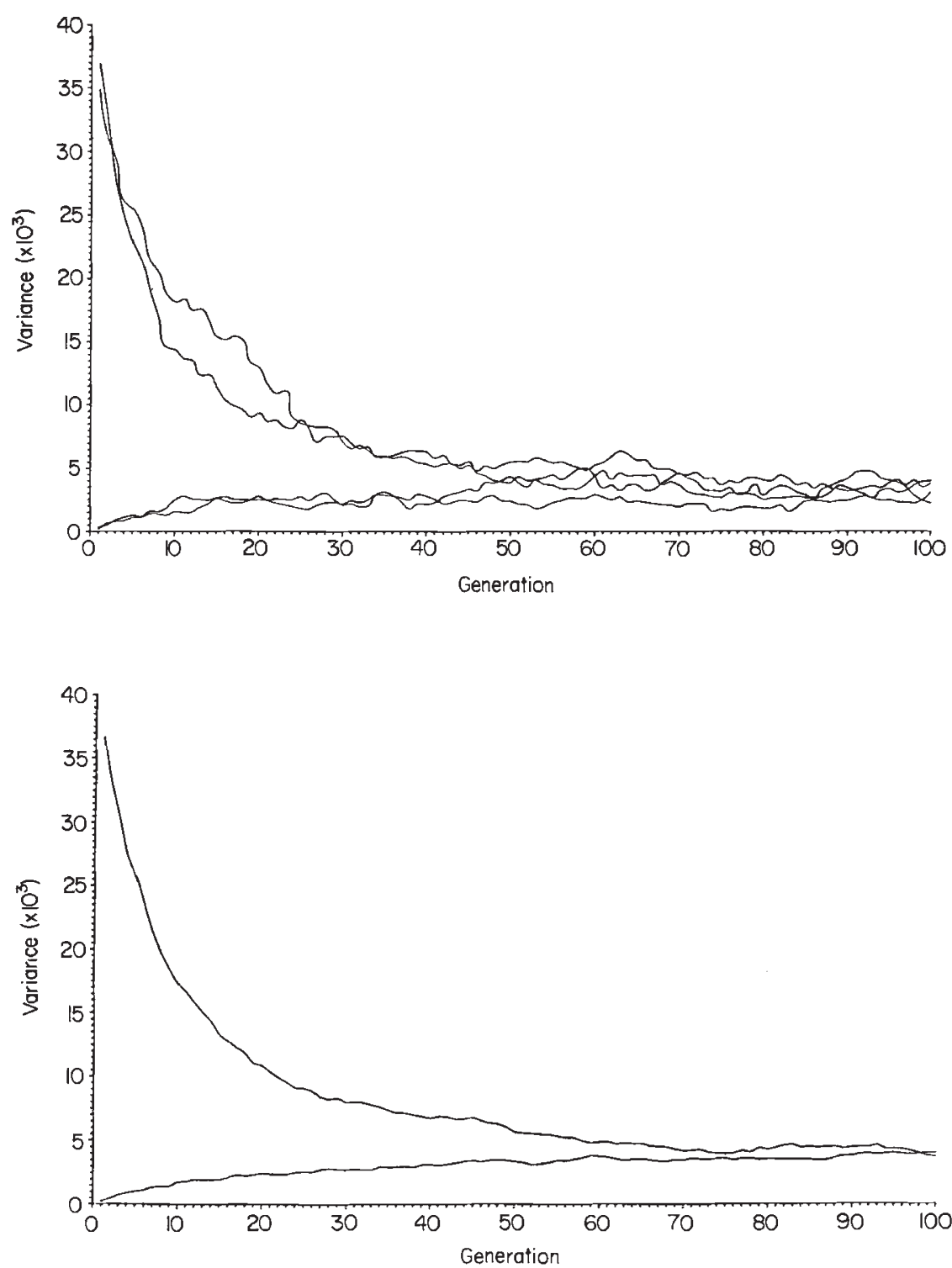

tive. The program was run ten times for 100 generations. No allele was lost in any of these runs, nor did any come near to being lost. The results obtained from two of these runs are shown in Fig. 1 (upper curves) and the average of the ten runs in Fig. 2 (upper curve), which show the variance of allele frequency against generation.

It is immediately apparent that though the inequality of allele frequency initially declines quite rapidly, it nevertheless takes many generations before stochastic equilibrium is attained. It is possible, however, that the approach to equilibrium has been impeded by starting these runs from a rather artificial population consisting of only 51 genotypes that have been replicated 73 times. Ten further runs were, therefore, carried out with an intial population consisting of eight replicates of each of the 465 possible genotypes in a population containing 31 equally frequent alleles, giving a population of size 3720 . The results obtained from two of these runs, shown in Fig. 1 (lower curves) and the average of the ten runs in Fig. 2 (lower curve), leave little doubt that the initial conditions have little or no effect on the time to equilibrium. In short, irrespective of whether we start with a population in which the allele frequencies are as unequal as they were in the R106 sample or one in which the frequency of every one of the 31 alleles is the same as that of every other, it takes somewhat between 70 and 80 non-overlapping generations of random mating in a population of a constant size of 3720 before the allele frequencies achieve stochastic equality. 


\section{Power of the modified $\chi^{2}$ test}

Detailed examination of the frequencies of individual alleles during the course of the runs described in the previous section revealed that even after a population had achieved equilibrium, the frequency of the most common allele in a particular generation could be as much as three times that of the least common allele. The magnitude of these inequalities is also evident from Figs 3-5 of Wright's (1939) paper. The question arises, therefore, whether the $\chi^{2}$ we have used to test the null hypothesis that the alleles are equally frequent in the populations from which the samples we have examined have been drawn (Campbell \& Lawrence, 1981b; Lawrence \& O'Donnell, 1981) is sufficiently powerful to detect inequalities in allele frequency caused by drift in populations at equilibrium. If so, we should, of course, have a very simple explanation of these inequalities and there would be no need to evoke any alternative explanation involving an extra effect of selection or a sampling effect over and above that due to drift. This question is of considerable importance to the work presented in this and preceding papers.

The power of the $\chi^{2}$ was tested empirically by drawing random samples of 51 plants, the size of the R106 sample, from a number of generations of simulated populations in which the allele frequencies, initially equal, had achieved equilibrium. At equilibrium, the variance of allele frequency in any one generation tends to be positively correlated with that in the next. After two generations, however, this correlation is small and after ten, neglible. Random samples of 51 plants were taken, therefore, from generations 120 , $130,140,150$ and 160 in each of a number of runs of three populations, the first of which was of size 3720 and contained 31 alleles, the second, a population of size 9300 also containing 31 alleles and the third, a population of 9320 individuals containing 40 alleles. The first of these populations is the same as one of those investigated in the previous section. The other two are of a size which is closer to that of the natural populations we have examined (see below) and the number of alleles in the third is probably closer to the number in these natural populations than is 31 . A representative portion of the results obtained from this investigation is shown in Tables 1-3.

While two of the twenty $\chi^{2}$ s in Table 1 are significant, one at the 5 per cent and the other at the 1 per cent level, none of the $30 \chi^{2}$ 's shown in Tables 2 and 3 are. Taken as a whole, these results are much as expected when the null hypothesis of equal allele frequency is true and this number of tests has been carried out. These results suggest that the test is not powerful enough to detect inequalities of allele frequency caused
Table 1 Simulation 1: a population of size 3720 containing 31 alleles. The number of alleles captured in samples of size 51 and $\chi^{2}$ 's testing the null hypothesis that the alleles are equally frequent in the population from which these samples have been drawn, the $\chi^{2}$ being calculated as:

$\chi_{\mid n-1\}}^{2}=(n-1) \frac{\left(\sum_{j=1}^{n} C_{j}^{2}-4 r^{2} / n\right)}{(2 r-4 r / n)}$,

where $n=$ number of alleles, $C_{j}=$ number of times the allele $S_{j}$ occurs and $r=$ number of plants in the sample

\begin{tabular}{rlc}
\hline Sample & $n$ & $\chi^{2}$ \\
\hline 1 & 30 & 24.735 \\
2 & 28 & 21.217 \\
3 & 30 & 26.563 \\
4 & 30 & 32.046 \\
5 & 31 & 35.964 \\
6 & 31 & 25.903 \\
7 & 29 & 23.364 \\
8 & 30 & 29.000 \\
9 & 30 & 35.092 \\
10 & 31 & 29.047 \\
11 & 29 & 26.902 \\
12 & 29 & 35.158 \\
13 & 28 & 30.910 \\
14 & 28 & 20.077 \\
15 & 30 & 29.000 \\
16 & 30 & 14.378 \\
17 & 30 & $43.016^{*}$ \\
18 & 29 & $55.207 * *$ \\
19 & 29 & 28.671 \\
20 & 28 & 29.199 \\
$* P=0.05-01 * * P$ & & \\
\hline \multirow{2}{*}{$* 01001$} & 0.001
\end{tabular}

Table 2 Simulation 2: a population of size 9300 containing 31 alleles

\begin{tabular}{rcc}
\hline Sample & $n$ & $\chi^{2}$ \\
\cline { 2 - 3 } 1 & 28 & 23.498 \\
2 & 29 & 17.467 \\
3 & 29 & 26.312 \\
4 & 30 & 30.219 \\
5 & 31 & 29.676 \\
6 & 30 & 31.437 \\
7 & 30 & 19.861 \\
8 & 29 & 23.954 \\
9 & 30 & 29.609 \\
10 & 30 & 21.080 \\
11 & 30 & 12.550 \\
12 & 29 & 14.519 \\
13 & 30 & 22.298 \\
14 & 31 & 24.016 \\
15 & 31 & 23.387 \\
\hline
\end{tabular}


Table 3 Simulation 3: a population of size 9320 containing 40 alleles

\begin{tabular}{ccc}
\hline Sample & $n$ & $\chi^{2}$ \\
\hline 1 & 37 & 26.659 \\
2 & 35 & 29.960 \\
3 & 37 & 29.405 \\
4 & 36 & 31.609 \\
5 & 38 & 26.118 \\
6 & 39 & 35.040 \\
7 & 35 & 28.546 \\
8 & 38 & 36.073 \\
9 & 35 & 27.131 \\
10 & 38 & 39.902 \\
11 & 37 & 36.867 \\
12 & 35 & 31.374 \\
13 & 37 & 27.166 \\
14 & 35 & 29.253 \\
15 & 37 & 20.451 \\
\hline
\end{tabular}

by drift alone in populations at equilibrium with samples of the size used in our experimental investigation of the polymorphism. This conclusion is consistent with Davies' (1981) comment that, because the power of the test is not very high, it is conservative and is thus unlikely to be misleading. On the other hand, the $\chi^{2}$ 's calculated on samples of 51 plants taken from the first ten generations of a population whose initial allele frequencies were the same as those of the R106 sample were virtually always significant (data not shown). Hence, we can be reasonably confident that the significance of the $\chi^{2}$ s calculated on the sample taken from the three natural populations we have examined (Table 3 of Lawrence et al., 1993) indicates inequalities of allele frequency that are greater than those caused by drift in populations at equilibrium.

\section{Discussion}

Having settled this important statistical question, we can consider the implications of our finding that it can take as many as $70-80$ generations for the allele frequencies in a population to achieve equilibrium after they have been disturbed, despite the fact that the selfincompatibility polymorphism is maintained by gene frequency-dependent selection.

There are three main points worth making about the results shown in Figs 1 and 2. Firstly, these preliminary results raise a number of questions concerning the effect of the number of alleles in a population and its size on the time to equilibrium. For example, do allele frequencies in a population containing only a small number of alleles reach equilibrium sooner than those containing a larger number? Again, is the time to equilibrium greater in large than in small populations containing the same number of alleles? These questions suggest that a systematic investigation of the joint effects of allele number and population size on the time to equilibrium by computer simulation would be worthwhile.

Secondly, these results confirm a point that we have made in previous papers, namely, that while the selective advantage of an allele is negatively related to its frequency in the population, it is also negatively related to the number of alleles present (Campbell \& Lawrence, 1981a; Lane and Lawrence, 1993; O'Donnell et al., 1993). In consequence, the strength of the frequency-dependent selection becomes progressively attenuated as the number of alleles in the population increases. There are two simple ways of demonstrating this effect. Firstly, we can calculate the percentage cross-incompatibility expected between plants in a population containing $k$ equally frequent alleles and, hence, $k(k-1) / 2$ equally frequent genotypes. Then the probability that an individual of genotype $S_{i} S_{j}$ receives pollen from another individual of the same genotype, assuming mating is at random, is the reciprocal of this quantity, that is, $2 / k(k-1)$. The expected percentage cross-incompatibility in populations containing from three to 200 equally frequent alleles is shown in Table 4. It is evident from this table that once a population contains 15 alleles, the percentage cross-incompatibility between individuals, the force which drives the frequency-dependent selection, is less than 1 per cent and that in a population containing 48 or more, the percentage cross-incompatibility is less than 0.1 per cent.

The second way of illustrating the attenuation of the frequency-dependent selection that maintains the polymorphism is to calculate the probability that a pollen grain carrying a particular allele, $S_{i}$, alights at random on the stigma of a plant containing the same allele. The number of genotypes in the population containing $S_{i}$ is $k-1$. Then assuming, once again, that both allele and genotype frequencies are equal, the proportion of stigmas on which an $S_{i}$ pollen grain in incompatible is this number divided by the total number of genotypes which is $2 / k$. The expected percentage pollen incompatibility in populations containing from three to 200 alleles is shown in Table 4. Although it is evident that percentage pollen incompatibility declines as the number of alleles in the population increases, the rate of attenuation from the point of view of the pollen, so to speak, is much less than it is from the point of view of the zygote. Thus, a population needs to contain more than 200 alleles for pollen incompatibility to fall below 1 per cent whereas only 15 are required to 
Table 4 Percentage cross-incompatibility between zygotes and pollen incompatibility for different numbers $(k)$ of equally frequent alleles

$k$ Cross-incompatibility (\%) Pollen-incompatibility $(\%)$

\begin{tabular}{rrr}
\hline 3 & 33.33 & 66.67 \\
4 & 16.67 & 50.00 \\
5 & 10.00 & 40.00 \\
6 & 6.67 & 33.33 \\
7 & 4.76 & 28.57 \\
8 & 3.57 & 25.00 \\
9 & 2.78 & 22.22 \\
10 & 2.22 & 20.00 \\
11 & 1.82 & 18.18 \\
12 & 1.52 & 16.17 \\
13 & 1.18 & 15.38 \\
14 & 1.10 & 14.29 \\
15 & 0.95 & 13.33 \\
16 & 0.83 & 12.50 \\
20 & 0.53 & 10.00 \\
24 & 0.36 & 8.33 \\
28 & 0.26 & 7.14 \\
32 & 0.20 & 6.25 \\
36 & 0.16 & 5.56 \\
40 & 0.13 & 5.00 \\
44 & 0.11 & 4.55 \\
48 & 0.09 & 4.17 \\
50 & 0.08 & 4.00 \\
100 & 0.02 & 2.00 \\
200 & 0.005 & 1.00 \\
\hline
\end{tabular}

achieve the same percentage incompatibility between zygotes.

These simple calculations are not intended to do more than illustrate the attenuation of frequencydependent selection as the number of alleles in the population increases. While there is no reason to doubt that Wright (1939) was aware of this negative relationship, we have been unable to find an expression in either this or his 1960 or 1964 papers that makes this explicit: an expression with the property would be welcome. A knowledge of the outcome of these calculations does, however, give some insight into the properties of the polymorphism. For example, to return for a moment to the first point made in this discussion, it is a reasonable guess that a population containing a small number of alleles would achieve equilibrium with respect to their frequency more quickly than one of the same size containing a larger number of alleles.

The third point worth making about these results concerns the time to equilibrium after allele frequencies have been perturbed. Thus, if some kind of environmental effect can cause allele frequencies to become unequal, there is no reason to suppose that, having occurred once, such an event will not occur again. But if this were the case, it is hardly reasonable to also suppose that its occurrence is so rare that it will not recur before the population has had sufficient time to achieve equilibrium after a previous perturbation, particularly if, as our results suggest, this can take as many as 70-80 generations. In short, can we expect any population of poppies to be in a state of equilibrium with respect to the frequencies of the alleles they contain?

The answer to this question obviously depends on our being able to identify one or more aspects of the population biology of the species which might be expected to cause inequalities of allele frequency greater than that caused by drift. It turns out that this task is far from easy. Thus, at first sight, $P$. rhoeas appears to be an opportunistic, colonizing species because it is found only on recently disturbed ground. Its populations, therefore, might be expected to vary greatly in size from one generation to the next. If this were the case, the effective size of the population would be much less than its actual size, so that opportunities for perturbation would be greater than in larger populations. The genetical evidence, however, is not consistent with this view of the population biology of the species. The fact that the populations we have examined appear to contain at least 32 alleles (Table 3 of Lawrence et al., 1993) suggests that their effective size, on Wright's (1939) calculations, is at least several thousand. Furthermore, if their size were to fluctuate to any great extent, populations would be expected to contain not only a smaller number of alleles than 32 but also a different complement of alleles from that of every other. But the evidence from our cross-classification experiments (O'Donnell et al., 1993) indicates that, on the contrary, the three British populations appear to contain essentially the same set of alleles.

There is, in fact, little difficulty in supposing that a stand of flowering plants consists of several thousands of individuals. Thus, a natural population found within $2 \mathrm{~km}$ of the R106 population turned out to contain 15,000 plants which, from the data obtained by scoring the seed produced by each of a random sample of 527 plants, gave an estimate of 7500 for the effective size of this population. The actual size of the population is much larger than this if account is taken, as it must, of the size of the seed bank in the soil by which populations persist from one flowering episode to the next. For example, the top $150 \mathrm{~mm}$ of soil in a second population of poppies we examined, close to the first, turned out to contain, on average, $3500 \mathrm{~m}^{-2}$ living but dormant seeds whereas the density of flowering plants in the first population was only $9 \mathrm{~m}^{-2}$. The size of these natural populations, therefore, when the zygotes in the seed bank are included, appears to be very much larger 
than the minimum size required, on Wright's theory (1939), to maintain the number of alleles they are estimated to contain. Hence, opportunities for any kind of sampling effect that might cause allele frequencies to become more unequal than is expected with drift alone appear to be rather limited in populations of this size. We note, in passing, that as the 'carrying capacity' of these populations is much greater than the number of alleles they contain, it is unlikely that any of these alleles will be lost by drift in the short to medium term at least.

There are three further points worth making about the reproductive biology of this species. First, since the seed of $P$. rhoeas can remain dormant in the soil for many years (Brenchley \& Warrington, 1933), it is likely that while most of the seed in the seed bank has originated from the immediately previous generation of flowering plants, some of it has come from one or more earlier generations. In these circumstances, a stand of flowering plants could include individuals that have germinated from the seed of several previous generations, so that the average length of a generation would be greater than the interval between flowering episodes and the overlap between generations would further retard the approach to equilibrium of allele frequencies.

Secondly, because the seed of this species can germinate successfully only on recently disturbed ground, the interval between flowering episodes in terms of years is likely to be both considerable and variable. Hence, it could well take two or three centuries for a population to go through 70-80 generations, so that if the allele frequencies in the populations we have investigated have, in fact, been perturbed by a single sampling event, this could have occurred very many years ago.

Thirdly, whereas the average number of seeds produced by the 527 plants of the first population was 7310 , the variance of seed output was no less than $5.472 \times 10^{7}$, giving a variance to mean ratio of 7485 . It is clear, therefore, that the distribution of progeny size in this population is far from random and that a minority of plants makes a much larger contribution of seed to the next generation than most. In consequence, the $S$-alleles of these plants will occur at a higher frequency in this generation than if the distribution of progeny size were random. While this can be expected to inflate the variance of allele frequency caused by drift, it is not clear that it could do so to an extent that would account for the unequal frequencies in the British populations. Nevertheless, this is a particularly intriguing possibility because it is quite different from the kind of perturbatory event we have had in mind so far. Whereas we have supposed that the latter occur only occasionally, any effect of non-random progeny size on the variance of allele frequency would be present in every generation and hence could result in a stable equilibrium of allele frequencies, provided that the variance to mean ratio of seed output did not vary drastically from one generation to the next. This, too, is a possibility that would be worth investigating by computer simulation.

\section{Acknowledgements}

In carrying out this work, S.O'D was supported by an SERC Research Studentship, M.D.L. by an NERC Research Studentship and D.F.M. and M.J.L. by an NERC Research Grant, all of which are gratefully acknowledged.

\section{References}

BRENCHLEY, W. E. AND WARRINGTON, K. 1993. The weed seed population of arable soil. II. Influence of crop, soil and methods of cultivation upon the relative abundance of viable seeds. J. Ecology, 21, 103-127.

CAMPBELl, J. M. AND LAWRENCE, M. J. 1981a. The population genetics of the self-incompatibility polymorphism in Papaver rhoeas. I. The number and distribution of $S$ alleles in families from three localities. Heredity, 46, 69-79.

CAMPBEll, J. M. AND LAWRENCE, M. J. 1981b. The population genetics of the self-incompatibility polymorphism in Papaver rhoeas. II. The number and frequency of $S$-alleles in a natural population (R106). Heredity, 46, 81-90.

CROSBY, J. L. 1966. Self-incompatibility alleles in the population of Oenothera organesis. Evolution, 20, 567-579.

DAVIES, P. 1981. A test of the equal frequency hypothesis for $S$-alleles. Appendix of Campbell, J. M. and Lawrence, M. J. (1981b).

EMERSON, s. 1939. A preliminary survey of the Oenothera organesis population. Genetics, 24, 524-537.

EWENS, W. J. 1964. On the problem of self-sterility alleles. Genetics, 50, 1433-1438.

EWENS, W. J. AND EWENS, P. M. 1966. The maintenance of alleles by mutation - Monte Carlo results for normal and selfsterility populations. Heredity, 21, 371-378.

FISHER, R. A. 1958. The Genetical Theory of Natural Selection, 2nd edn. Dover Publications, New York.

KIMURA, M. AND MARUYAMA, T. 1966. In: Mayo, O. (1966).

LANE, M. D. AND LAWRENCE, M. J. 1993. The population genetics of the self-incompatibility polymorphism in Papaver rhoeas. VII. The number of S-alleles in the species. Heredity, 71, 596-602.

LAWRENCE, M. J. AND FRANKLIN-TONG, V. E. 1994. The population genetics of the self-incompatibility polymorphism in Papaver rhoeas. IX. Evidence of an extra effect of selection acting on the $S$-locus. Heredity, 72, 353-364.

LAWRENCE, M. J., LANE, M. D., O'DONNELL, S. AND FRANKLIN-TONG, V. E. 1993. The population genetics of the self-incompatibility polymorphism in Papaver rhoeas. V. The cross-classifi- 
cation of the S-alleles of samples from three natural populations. Heredity, 71, 581-590.

LAWRENCE, M. J. AND O'DONNELL, S. 1981. The population genetics of the self-incompatibility polymorphism in Papaver rhoeas. III. The number and frequency of $S$ alleles in two further natural populations (R102 and R104). Heredity, 47, 53-61.

MAYO, o. 1966. On the problem of self-incompatibility alleles. Biometrics, 22, 111-120.

MORAN, P. A. P. 1962. The Statistical Processes of Evolutionary Theory. Clarendon Press, Oxford.

o'DONNELL, S., LANE, M. D. AND LAWRENCE, M. J. 1993. The popu- lation genetics of the self-incompatibility polymorphism in Papaver rhoeas. VI. The estimation of the overlap between the allelic complements of a pair of populations. Heredity, 71, 591-595.

WRIGHT, s. 1939. The distribution of self-incompatibility alleles in populations. Genetics, 24, 538-552.

WRIGHT, s. 1960 . On the number of self-incompatibility alleles maintained in equilibrium by a given mutation rate in a population of given size: a re-examination. Biometrics, 16, 61-85.

WRIGHr, s. 1964 . The distribution of self-incompatibility alleles in populations. Evolution, 18, 609-619. 